\title{
La historiografia de la Revolución Rusa, cien años después
}

\author{
Stephen A. Smith ${ }^{1}$
}

Universidad de Oxford

stephen.smith2@history.ox.ac.uk

El centenario de la Revolución Rusa ofrece una buena ocasión para preguntarnos de qué modo la historiografia reciente contribuye a nuestra comprensión sobre ese trascendental acontecimiento.Vivimos en una época que no es especialmente afin a la idea de revolución. En el mundo occidental, el alcance de la política se ha reducido desde la década de 1970, con la aparición del neoliberalismo, el colapso del comunismo, el incremento de la preocupación por los derechos humanos y los límites de una política definida por el libre mercado, la buena administración y los derechos individuales. Las referencias a la "revolución" no han desaparecido del todo, pero se trata, en palabras de Arno Mayer, de "la celebración de revoluciones esencialmente pacíficas en pro de los derechos humanos, la propiedad privada y el capitalismo de mercado" (2001: 3). Se podría añadir que las revoluciones "de colores" del este europeo y el Cáucaso, así como las de la primavera árabe, difícilmente hayan sido buenos modelos para aquellos que pretenden efectuar un cambio político por medios violentos. El relevamiento historiográfico que sigue se enmarca en este contexto. Considero que, si bien nuestro conocimiento sobre la Revolución Rusa y la guerra civil se ha incrementado en forma significativa, en muchos aspectos decisivos ha disminuido nuestra capacidad para comprender -y ciertamente para empatizar con- las aspiraciones de 1917.

Este artículo busca identificar las tendencias y problemas analíticos que han ocupado a los historiadores desde, aproximadamente, los inicios del siglo XXI. La atención se centra en el período que se extiende desde 1914 hasta la consolidación de un nuevo orden soviético, en 1922, y no únicamente en las dos revoluciones de 1917. El ensayo comienza

1. Publicado originalmente en inglés en Kritika: Explorations in Russian and Eurasian History, vol. 16, $\mathrm{n}^{\circ}$ 4, otoño de 2015 (nueva serie), págs. 733-749. Traducción: Antonio Oliva y Lucas Poy.

(Archivos, año VI, $\mathrm{n}^{\circ} 11$, septiembre de 2017, pp. 79-98) 
bosquejando ciertas tendencias interpretativas que han tenido influencia en la historiografia reciente y rastreando algunos de los temas que han atraído el mayor interés académico. No tiene una pretensión de integralidad: en particular, no intenta abordar el enorme volumen de estudios sobre las regiones no rusas del imperio ni discutir las abundantes y excelentes colecciones de material de primera mano que han aparecido, especialmente en Rusia. ${ }^{2}$

$$
* * *
$$

En la primera década post-soviética -la de 1990-, tuvo lugar la apertura de los archivos y un intenso debate público, en la Federación Rusa, sobre el significado de la era soviética para el futuro del país. Entre los historiadores profesionales existía un vigoroso rechazo a los estereotipos ideológicos que habian estructurado la historiografia de la era soviética, centrados en el mito de la Gran Revolución Socialista de Octubre, así como un apremio por investigar "puntos ciegos" y temas hasta entonces prohibidos. Desde comienzos del siglo XXI, el debate público en Rusia acerca de la era soviética se ha apaciguado un poco, pero de ninguna manera ha desaparecido. Entre los historiadores profesionales están floreciendo investigaciones detalladas, que se basan en la nueva documentación; la gama de temas en estudio se ha ampliado y el tono del intercambio académico se ha vuelto más desapasionado. Entre los estudiosos occidentales, que trabajan principalmente en los Estados Unidos, en Alemania y en el Reino Unido, el volumen de investigaciones históricas sobre la revolución y la guerra civil se ha reducido en comparación con las décadas de 1970 y 1980. La apertura de los archivos en la década de 1990 provocó una reorientación hacia los años de Stalin -el periodo sobre el cual los historiadores menos conocian-, quitando así fuerza a los estudios sobre la revolución, una etapa acerca de la cual la base de fuentes nunca había sido tan exigua como para el período posterior a 1921. Además, la disminución del interés por la historia social en el mundo académico -y en especial por la historia del trabajo- tuvo el efecto de reducir la cantidad de investigaciones realizadas sobre 1917, muchas de las cuales habian estado animadas por el interés en la "revolución desde abajo". Por último, la marginalización de la izquierda política a nivel internacional, tras el colapso del comunismo y el auge del neoliberalismo, creó un clima en el que las revoluciones ya no eran vistas con demasiada simpatía, y en el cual los historiadores se interesaban menos por mostrar "lo que falló" en la revolución bolchevique que por

2. Hay fuentes fundamentales para el estudio de la dinámica política de 1917 que aún podrian estar por descubrirse. Ver Lyandres (2013). 
demostrar que una revolución minoritaria conduce en forma inevitable a una dictadura totalitaria. A pesar de esto, los estudios occidentales sobre la revolución de ninguna manera se han agotado: algunos de los mejores trabajos se señalan a continuación.

Una de las tendencias más significativas en la historiografia del nuevo siglo ha sido posicionar plenamente a las revoluciones de 1917 en una narrativa que comienza con el estallido de la guerra en 1914 y termina con el establecimiento de la Unión Soviética en $1922 .{ }^{3}$ La historiografia, por supuesto, siempre reconoció que la derrota militar, el cansancio de la guerra y la creciente privación en el frente interno fueron causas principales de la caída de la monarquía y del posterior fracaso del gobierno provisional. Pero los historiadores soviéticos, en particular, prestaban poca atención a la Primera Guerra Mundial en sí misma, a no ser como prolegómeno de la revolución. La historiografia reciente, tanto en Rusia como en el mundo occidental, y sobre todo los trabajos que encuadran la historia de Rusia en un marco imperial, explora de qué modo las fuerzas sísmicas desatadas por la guerra transformaron el panorama político en gran parte de Europa, en un proceso que duró desde 1917 hasta alrededor de 1923, y cómo la guerra total -una noción un tanto nebulosa- alumbró instituciones y prácticas políticas que esbozaron las del régimen soviético. ${ }^{4}$

En 1994, publiqué un artículo un tanto incómodo que lamentaba la falta de voluntad de los historiadores rusos para hacer frente a los desafíos teóricos del posmodernismo y el "giro cultural", argumentando que la mera incorporación a paradigmas establecidos de franjas de material de archivo recientemente disponible no revitalizaba el conocimiento académico (Smith, 1994). Argumenté que el reto consistía en tomar en serio los diversos enfoques agrupados crudamente como "posmodernos", con el fin de estimular la reflexión sobre el modo en que, durante la revolución, movimientos sociales, partidos políticos, organizaciones de base, líderes carismáticos e ideologias radicales pusieron en circulación nuevas prácticas discursivas, cuyo resultado fue convencer a millones de personas comunes de poder entenderse a sí mismos, a sus intereses y a su predicamento de nuevas maneras, es decir, poner en primer plano ciertas identidades y marginar otras (Smith, 2008). Desde esta perspectiva, la dinámica política de la Revolución Rusa no

3. Holquist (2002), Sanborn (2003). Ver también la serie Russia's Great War and Revolution, 1914-1922, en progreso de publicación. En el momento de la escritura de estas lineas, han aparecido tres volúmenes: Lohr, Tolz, Semyonov y von Hagen (2014) y Frame, Kolonitskii, Marks y Stockdale (2014, dos tomos). Se publicarán cinco volúmenes más.

4. Para una argumentación interesante que plantea que el principal efecto de la guerra era lograr una "descolonización del imperio ruso", ver Sanborn (2014). 
se trata solamente de quién hizo qué y cómo, sino del modo en que las personas se entendieron a sí mismas y buscaron orientarse durante tiempos profundamente inquietantes. Aunque sigue siendo pequeño el número de historiadores de la Revolución Rusa que se involucran directamente con la teoría social, el impacto del "giro cultural", si no del posmodernismo en toda la línea, ha sido significativo. Boris Kolonitskii ha estudiado de qué modo los símbolos -tales como el águila de dos cabezas, la "Marsellesa" o la bandera roja- han actuado como objetos de confrontación política que contribuyeron a delimitar el terreno de la lucha política (Kolonitskii, 2001; Figes y Kolonitskii, 1999: 30). Más recientemente, en un trabajo maravillosamente original, ofreció un análisis sociocultural densamente trabajado acerca de la forma en que la unidad entre el zar y el conjunto de la población se fue erosionando rápidamente en todos los niveles de sociedad (Kolonitskii, 2010). Una de sus líneas de investigación se centra en mostrar cómo la autoridad sagrada de la familia real se vio debilitada por los rumores, en especial aquellos relacionados con Alexandra Fedorovna. El de los rumores, de hecho, ha sido uno de los campos de trabajo más fértiles de los estudiosos rusos -no sólo historiadores de la cultura, sino también antropólogos y psicólogos sociales- en la época post-soviética. ${ }^{5}$ Mark Steinberg (2001), quizás el historiador más receptivo a los enfoques posmodernos, ha iluminado la relación que existe entre la experiencia social, las estrategias de escritura y la voz del autor, en un ensayo pionero sobre los lenguajes de la revolución: en él demuestra cómo, en 1917, la gente del común articuló su experiencia de la guerra y la revolución a través de un lenguaje cargado de emoción y fervor moral.

En Rusia, un desarrollo paralelo fue el que a veces se conoce como "giro antropológico", un vuelco de la atención que se aleja de los militantes revolucionarios, los partidos y las ideologias y se acerca a la vida diaria y a las personas comunes. Sergei Iarov (2006) exploró el modo en el cual las personas se adaptaron al lenguaje, los estereotipos y las reglas del juego del nuevo orden soviético, centrándose en las formas de argumentación, la politización de los discursos y del ocio, la cooperación entre los intelectuales y el gobierno y la retracción de la protesta laboral. Oksana Nagornaia (2010) reconstruyó la experiencia de los soldados y oficiales rusos que se convirtieron en prisioneros de guerra en Alemania, mostrando cómo crearon espacios limitados de autonomía, aun cuando estaban divididos por factores como el grado de contacto con la sociedad civil, el origen étnico, la política y los privilegios diferenciales. Un énfasis similar en la experiencia de la vida diaria se evidencia en el

5. Narskii et al. (2011). Sobre los rumores relacionados con la traición, ver Fuller (2006: 43-48). 
estudio realizado por O.M. Morozova (2010) sobre los soldados del Ejército Rojo y los partisanos rojos durante la guerra civil. ${ }^{6}$ Su trabajo utiliza biografias escritas entre 1920 y 1935 por veteranos que solicitaban pensiones, con el objetivo de observar cómo utilizaban los relatos de su experiencia durante la guerra para reclamar prestaciones de asistencia social. En un estudio más amplio, explora la manera en que la formación personal y la psicología dieron forma a las estructuras y las prácticas de los ejércitos rojo y blanco durante la guerra civil, aunque parecen discutibles algunas de sus generalizaciones acerca de la importancia de los procesos psicológicos para provocar el desorden y la transformación revolucionaria (Morozova, 2012). Estas obras ponen en evidencia que el interés por la psicologia social ha sido una caracteristica particular de la historiografia reciente en Rusia.

Esta preocupación por la psicología de masas se basa en un interés por el tema que se desarrolló en los últimos años del régimen soviético (algo que no tiene un paralelismo entre los historiadores sociales occidentales, que analizan los movimientos de masas en términos de cálculo racional y normas conscientes). V.P. Buldakov (1997) entiende que los procesos "psico-mentales" fueron los que alimentaron la caída en el caos revolucionario. Interpreta el período 1914-1921 como un segundo smuta, un "tiempo de problemas", provocado por el hecho de que la Primera Guerra Mundial desacralizó el poder real al mismo tiempo que sacralizó la violencia. Con la revolución de febrero, toda restricción ética fue dejada de lado y se dio vía libre a los lúmpenes más agresivos. ${ }^{7}$ Buldakov sostiene que, en todos los niveles de la sociedad, hubo un resurgimiento de un comunalismo atávico (obshchinnost') que jugó en favor de los bolcheviques, quienes tuvieron éxito no porque se presentaran como campeones de los intereses populares sino porque proyectaron una imagen que concordaba con la psicología de las masas. Es una idea interesante, pero sus generalizaciones acerca de la psicología de masas son muy drásticas, y su imagen de la población como una chusma que anhela el dominio político es muy parecida a la difundida por Hippolyte Taine en relación con la Revolución Francesa.

En los Estados Unidos, podemos observar una apropiación llamativa del posmodernismo en la obra de un grupo de estudiosos, a veces conocido como "escuela de la modernidad", que se basó en los trabajos de Michel Foucault sobre la gobernabilidad y la biopolitica. Interpretan el régimen soviético como una variante de la modernidad europea, llamando la atención sobre elementos tales como la planificación, el

6. Para una estimulante revisión de libros recientes sobre la experiencia en Austria, Alemania y otros prisioneros de guerra en Rusia, véase Gatrell (2005).

7. El tema continuó en Buldakov (2007 y 2010). 
estado de bienestar, la valoración de la ciencia, las formas de vigilancia social, la gobernabilidad y las "disciplinas de la personalidad", elementos supuestamente comunes tanto al régimen soviético como a regimenes políticos radicalmente distintos de la Europa de entreguerras (Hoffmann y Kotsonis, 2000; Hoffmann, 2011).Suelen plantear que la especificidad del régimen soviético residia en su uso informal y extendido de la violencia como técnica de gobierno. Este énfasis en la violencia ha sido una preocupación central en buena parte de los trabajos recientes sobre la Revolución Rusa. Los teóricos clásicos del totalitarismo bolchevique veían a la violencia como un resultado de la ideología y de la falta de legitimidad politica del régimen; la escuela de la modernidad, por el contrario, localiza sus raíces en las prácticas de clasificación, recopilación de información, control, encarcelamiento y expulsión comunes a los diferentes estados modernizadores europeos (Holquist, 2003). Desde esta perspectiva, la Primera Guerra Mundial constituyó un punto de inflexión que llevó a una masiva expansión y militarización de las prácticas diseñadas para dar forma al "cuerpo social", que tenían sus origenes en el siglo XIX. El impresionante estudio de Peter Holquist sobre la Revolución y la Guerra Civil en la región del Don, por ejemplo, considera que la violencia bolchevique no solo fue consecuencia del impulso por suprimir enemigos políticos sino también de una aspiración por crear una sociedad purgada de elementos contaminantes, en particular durante el breve episodio de la "des-cosaquizacion" (Holquist, 2002, capítulo 6). Sin embargo, su énfasis en la violencia "escisional" -o sea, la violencia diseñada para eliminar grupos específicos que eran percibidos como socialmente perjudiciales o políticamente peligrosos para el cuerpo social- parece más apropiado para la era de Stalin que para un período en el cual los bolcheviques tenían dificultades para establecer el monopolio de la violencia. En una línea similar, Joshua Sanborn afirma que la práctica de la violencia era la "precondición esencial para ser un ciudadano y un hombre" entre 1905 y 1925, aunque nunca resuelve el problema, que él mismo identifica, de cómo conciliar la disposición de los bolcheviques por usar el terror con su simultáneo deseo de contener la violencia. ${ }^{8}$

8. Sanborn (2003: 200) cita un pasaje de Handbook of the Red Army Soldier, publicado en 1918 para apoyar su afirmación de que la justificación de la violencia bolchevique era una "glorificación de la sangre". Sin embargo, en el inicio el Handbook justifica la violencia en términos convencionales como necesaria para crear un mundo mejor: "“¿Quién eres tú, camarada?" [...] "Soy un defensor de los trabajadores y los pobres de todo el mundo." "¿Por qué estás luchando?" [...] "Por justicia [pravda]. Para que las tierras y fábricas, y los ríos y bosques, y toda la riqueza pertenezca a las personas que trabajan"” (Partiino-politicheskaia rabota v Krasnoi Armii: Aprel' 1918-fevral' 1919. Dokumenty [Moscow: Voennoe izdatel'stvo, 1961], 44). 
También los especialistas alemanes han tendido a ver la violencia como la modalidad principal de instalación del régimen soviético, aunque suelen ser escépticos respecto a la conceptualización, realizada por la escuela de la modernidad, de la violencia politica como elemento constitutivo en un modelo generalizado de "modernidad". Jörg Baberowski cuestiona que la ideología tenga mucho que ver con el impulso hacia la violencia, argumentando que la violencia tiene lugar porque las personas aprovechan la oportunidad de usarla para lograr sus objetivos cuando pueden hacerlo, y una vez que la violencia se ha iniciado, no hay más alternativa que utilizarla para sobrevivir o sucumbir a ella (2012: 476). Los estudios alemanes se destacan por su preocupación por diferenciar distintos tipos de violencia, prestando atención a sus distintas formas y a sus significados y efectos sociales diferenciales. Se basan en el trabajo de sociólogos y filósofos alemanes que han categorizado los modos en que la experiencia de los daños corporales se convierte en significativa. Felix Schnell (2012), por ejemplo, hace hincapié en las funciones asociativas $\mathrm{y}$ formativas de la identidad que puede tener la violencia, observando la violencia colectiva del otomany en Ucrania como un acto comunicativo que tenía tanto que ver con estrechar lazos entre los protagonistas como con aterrorizar a los enemigos. En términos generales, los historiadores alemanes se acercan a la violencia no sólo en términos instrumentales, como un medio para consolidar el poder, sino también como un mecanismo que crea, dramatiza, y desafia las jerarquias del poder.

El estimulante estudio de Joshua Sanborn sobre la conscripción en Rusia (2003) explora cómo el servicio militar se convirtió en un deber nacional y de qué modo la propia identidad nacional estaba militarizada. Su trabajo es expresión de la tendencia a reubicar la Revolución Rusa en un marco nacional e imperial antes que en uno de clase. También Eric Lohr (2003) hace hincapié en los efectos nacionalizadores de la guerra, en una obra que estudia de qué modo los "extranjeros enemigos" -hombres de negocios y agricultores alemanes, judios, musulmanes y otros- fueron sometidos a la deportación, la internación y la expropiación. Sostiene que la ampliación, en tiempos de guerra, de las prácticas de gobernabilidad relevadas por la escuela de la modernidad y, en particular, el intento de expulsar a las minorias étnicas del cuerpo político, eran en realidad un reflejo de la debilidad del nacionalismo ruso. El debate acerca de la relación entre nación e imperio, abierto por primera vez en la década de 1990 por historiadores como Geoffrey Hosking (1997), ha sido particularmente fructífero a la hora de promover investigaciones sobre la guerra y la revolución en el marco del Imperio. Actualmente, resulta imposible comprender la Revolución Rusa si no es en el contexto de un imperio multiétnico que buscaba desesperadamente competir con otros tres imperios en los frentes oriental y del Cáucaso. A partir de la 
revolución de febrero, por otra parte, el curso de la revolución estuvo intimamente conectado con las luchas de los pueblos no rusos del Báltico, las fronteras occidentales, el Cáucaso y Asia Central para lograr diferentes grados de autodeterminación nacional o étnica. Sin embargo, en lo que respecta a la política de 1917 como tal, podríamos correr el riesgo de perder de vista que una peculiaridad de la Revolución Rusa -en comparación con revoluciones posteriores en China, Yugoslavia, Vietnam o Cuba- fue la primacía de la clase como marco dominante en el que se libró la lucha política, algo que no implica negar que las identidades nacionales, con frecuencia sobredeterminadas por la clase, tuvieran cada vez más influencia (Smith, 2006).

En los últimos años aparecieron excelentes estudios sobre la revolución en las provincias. Sin embargo, cabe mencionar en primer lugar el trabajo de Alexander Rabinowitch (2007) sobre el primer año de gobierno bolchevique en Petrogrado, una refinada secuela de su clásico estudio sobre la toma del poder en octubre. Su obra refleja los modos en que las crisis política, militar y económica de 1918 transformaron el poder soviético en un régimen de partido único, un desafio al resurgimiento de la tendencia que deja de lado la contingencia en favor de un determinismo ideológico u organizativo. En cuanto a la revolución en las provincias, los historiadores soviéticos sufrian la presión de tener que demostrar que se trataba, en lo esencial, de variantes de un proceso revolucionario uniforme -el inexorable avance del "poder soviético"- lo cual no quita valor a algunos de los mejores trabajos, como el estudio de Grunt (1976) sobre Moscú o el de Gerasimenko (1972) sobre el Bajo Volga. Los estudios más recientes sobre la revolución en las provincias, de todas formas, desafian esta concepción y ponen en cuestión la idea de que la toma del poder bolchevique en Petrogrado haya sido el quiebre decisivo con el viejo orden. En realidad, la lucha para establecer el poder soviético en los pueblos y ciudades de la Rusia europea y de Siberia comenzó recién después de octubre y en muchas regiones continuó, con avances y retrocesos, hasta 1920. Sarah Badcock (2007), en un estudio comparativo de dos provincias del Volga, Nizhni Novgorod y Kazan -la primera étnicamente rusa y relativamente industrializada, la segunda étnicamente mixta y económicamente menos desarrollada-, demuestra la importancia de las conexiones locales a la hora de establecer patrones políticos en dos ciudades donde los partidos organizados eran débiles. En su ampliamente documentado estudio sobre la Guerra Civil en la provincia de Saratov, Donald Raleigh (2002) muestra de qué modo la corrupción y la ilegalidad caracterizaron a los soviets y a las organizaciones partidarias desde el principio. ${ }^{9}$ Mientras que los traba-

9. Ver también Raleigh (1986). 
jos centrados en Petrogrado hacen hincapié en la fuerte conexión que existia entre el partido bolchevique y los movimientos de soldados y obreros radicalizados, este vínculo se revelaba mucho más tenue fuera de la capital, incluso en Moscú. En las provincias, los significados y los resultados de la revolución se conformaron a partir de una variedad de factores ecológicos, socioeconómicos, étnicos, y políticos (Narskii, 2001; Posadskii, 2010; Penter, 2000). En qué medida esto justifica la idea de un caleidoscopio de revoluciones, sin embargo, sigue siendo una pregunta abierta (Retish, Badcock y Novikova, 2015). Es discutible que la variación regional en la Revolución Rusa haya sido considerablemente menor que en las revoluciones mexicana o china.

Es, por supuesto, la ausencia de las masas rurales en Petrogrado lo que hace a los episodios de la capital tan atípicos respecto a la revolución en su conjunto. Una vez más, los estudios recientes ofrecen representaciones matizadas de la revolución en el campo. Si en la región central de la Tierra Negra fue la comuna la fuerza impulsora del movimiento por la apropiación de tierras pertenecientes a la nobleza terrateniente (y a campesinos que se habian separado de la comuna), en otras regiones la redistribución de la tierra fue un proceso a menudo coordinado por los departamentos de tierras del distrito y por los soviets locales. En el caso de Viatka, donde la comuna se revitalizó durante la guerra, Aaron Retish muestra de qué manera las intervenciones del nuevo Comisariado de Agricultura y de los departamentos de tierras de los soviets locales frenaron la distribución espontánea de tierra impulsada por las comunas individuales (2008: 161). En Arkhangel'sk, menos de una cuarta parte de las comunas habian llevado a cabo la redistribución de la tierra cuando la provincia quedó en poder de los blancos, en noviembre de 1918 (Sablin, 2009). En Moscú, gran parte de la tierra se colocó "a cuenta" (na uchet) del departamento de tierra del soviet provincial, de acuerdo con la Ley Básica de Socialización de la Tierra, del 17 de enero de 1918, y fue redistribuida con la participación de los soviets locales (Kovalev, 2007). En términos generales, sin embargo, y dado que la formación de los soviets municipales fue lenta, los soviets de distrito y de provincia fueron a menudo incapaces de ejercer mucha influencia a nivel de las bases. En Smolensk, por ejemplo, sólo 33 de un total de 239 soviets municipales habian creado departamentos de tierras en abril de 1918 (Hickey, 1996). Si bien la imagen habitual de la revolución agraria como un movimiento espontáneo sigue siendo precisa -lo que nuevamente distingue a Rusia de otras revoluciones comunistas posteriores- la dinámica entre redistribución de la tierra y naciente formación del Estado reclama mayores investigaciones.

Todavía sabemos mucho menos sobre el destino de la élite rusa que sobre el de sus clases populares. Los historiadores rusos, tanto en la 
época soviética como en la post-soviética, han hecho un buen trabajo sobre las fortunas socioeconómicas de la nobleza terrateniente y, más recientemente, sobre su orientación política a finales del periodo zarista (Bibin, 2000; Kir'ianov, 2001). Sin embargo, poco se ha hecho sobre su destino en 1917. Existen dos libros de historiadores occidentales que contribuyen a llenar ese vacío: Matthew Rendle (2009) ofrece un estudio sutil sobre los terratenientes, la nobleza, y los oficiales donde demuestra que de ninguna manera fueron una fuerza pasiva durante la agitación revolucionaria, si bien la pérdida de sus privilegios y las expropiaciones de sus propiedades exacerbó su desarraigo político y social. Douglas Smith (2012: 7) advierte que "la nobleza habia producido generaciones de escritores, artistas y pensadores, de académicos y científicos, tanto reformadores como revolucionarios", pero su nostálgico estudio de los Golitsyns y Sheremetevs es tipico de una muy popular historiografia que coloca a la nobleza como víctima inocente de un malvado régimen bolchevique. Sin embargo, no se pregunta cuántos terratenientes se manifestaron en defensa de la propiedad en 1917 ni por qué los campesinos se levantaron contra ellos. Nos dice que "la tragedia del destino de los nobles prefiguró futuras atrocidades del siglo XX... desde la Alemania de Hitler hasta la Camboya de Pol Pot" (2012: 8): una mirada de la Revolución Rusa que suena demasiado familiar, en la cual los bolcheviques aparecen como los únicos responsables de los trágicos sucesos posteriores.

El interés académico por la resistencia campesina durante la Guerra Civil se convirtió en un tema importante de la historiografia post-soviética en la década de 1990, y continúa siéndolo hasta hoy (Fatueva, 1996; Iarov, 1999; Kondrashin, 2001). La rebelión de Antonov en Tambov, que se extendió desde el otoño de 1920 hasta la primavera de 1921, es el objeto de un agudo estudio de Erik Landis, quien combina la investigación empírica con la teoría de los movimientos sociales (Landis, 2008; DuGarm, 2001). Si bien es la más conocida, la rebelión de Antonov fue sólo una entre muchas: la eliminación de la amenaza blanca y el endurecimiento de la requisa de alimentos, en el invierno de 1920, impulsaron la transformación de émeutes de pequeña escala en levantamientos de masas, con más de 50 rebeliones que irrumpieron en lugares tan lejanos como el Don, Kuban, Ucrania, el norte del Cáucaso, Asia central, Bielorrusia y Karelia. La mayor rebelión, en términos de número y escala geográfica, estalló en el oeste de Siberia, donde los campesinos, con el respaldo del movimiento partisano antiblanco, se volvieron contra los rojos después del comienzo de la implacable requisa de alimentos en el verano de 1920. En su punto más alto, el levantamiento involucró al menos a 100.000 hombres (casi el mismo tamaño que el ejército del almirante Kolchak), en una superficie de 1,5 millones de kilómetros cuadrados (Shishkin, 
2000). Todas estas rebeliones tenían raíces en privaciones económicas, pero también estaban motivadas por la profunda hostilidad hacia un régimen que no sólo era duro, sino también más corrupto a nivel local de lo que alguna vez se consideró. Entre los insurgentes, sin embargo, no existia un programa unificado -o un mando unificado, en el caso de Siberia occidental- más allá de un deseo por preservar "el poder soviético" purgado de comunistas. En ese sentido, aunque ciertamente eran antibolcheviques, no eran rebeliones contrarrevolucionarias.

Debido a su enorme magnitud, la rebelión campesina representaba una amenaza mucho mayor para el régimen bolchevique que la rebelión de Kronstadt. Sin embargo, es esta última la que se apoderó de la imaginación de generaciones ansiosas por comprender "lo que salió mal" después de los dias de gloria de 1917. ¿Los rebeldes estaban llevando adelante un último intento desesperado por defender los ideales de la revolución? ¿O estaban amenazando con derrocar al régimen que representaba la única defensa de la misma? Lo que resulta incongruente es que la apertura de los archivos haya sido incapaz, en gran medida, de resolver este problema. El documento más importante aparecido después de 1991 fue un informe del 5 de abril 1921 firmado por Ia. S. Agranov, un plenipotenciario del Departamento Especial de la Cheka, ${ }^{10}$ que caracterizaba la rebelión como "un levantamiento desorganizado de la masa de marineros y obreros" y negaba que los rebeldes tuvieran ninguna conexión con las fuerzas blancas. Fue ése el documento que respaldó el perdón presidencial de Boris Yeltsin a los rebeldes, el 10 de enero de 1994. La primera colección de documentos sobre Kronstadt, que incluía este informe, destacaba la conexión existente entre la rebelión y las huelgas por motivos económicos que habian tenido lugar en la capital, y parecía refutar la acusación hecha por los bolcheviques de los rebeldes como "contrarrevolucionarios" (Naumov y Kosakovskii, 1997). Sin embargo, una segunda colección de más de 800 documentos, aparecida en 1999, proporcionó una extensa documentación sobre la participación de contrarrevolucionarios en la rebelión (aunque con poco sentido de su eficacia), que pretendia mostrar el papel fundamental desempeñado por la Organización Militar de Petrogrado, dirigida por el profesor V.N. Tagantsev. La supresión de esta organización fue acompañada por la detención de 833 personas, 96 de las cuales fueron ejecutadas o murieron en cautiverio (Vinogradov y Kozlov, 1999). La introducción insinúa oscuramente que el líder de los marineros, S. M. Petrichenko, estaba en connivencia con los blancos desde el principio.

10. La Cheka era la "Comisión Extraordinaria Panrusa para la lucha con la Contrarrevolución y el Sabotaje”, la primera versión de la policía política soviética. 
Irónicamente, la entrega de toda la documentación sirvió para generar más polémica antes que para establecer cierta claridad.

Otra línea de investigación que ha continuado desde la década de 1990 es la que estudia la oposición obrera a los bolcheviques durante la Guerra Civil. Desde hace tiempo se sabe que en la primavera de 1918 se desplomó el apoyo de los trabajadores y que revivió la suerte de los mencheviques y los eseristas, debido a su determinación por mantener elecciones democráticas en los soviets y en los sindicatos. ${ }^{11}$ Con el inicio de la guerra civil a gran escala, el apoyo obrero a los bolcheviques volvió a crecer, aunque esto no impidió frecuentes protestas por las raciones inadecuadas y la disciplina laboral (Churakov, 2004). Trabajos recientes, sin embargo, han explorado un tema que era tabú en la época soviética: el alcance del apoyo dado por los trabajadores a los blancos. En los Urales, donde los trabajadores de la industria metalúrgica y metalmecánica habian constituido el embrión de la clase obrera rusa, tuvieron lugar 37 levantamientos contra los bolcheviques entre diciembre de 1917 y julio de 1918 (Porshneva, 2013). Con diferencia, el más significativo se produjo en el otoño de 1918, en los talleres de armamento Izhevsk y Votkinsk, que en conjunto empleaban a más de 30.000 trabajadores. En Izhevsk, la Guardia Roja local, bajo el control de los eseristas maximalistas, perdió el apoyo de los trabajadores debido a las duras requisas y las detenciones arbitrarias, así como a la prohibición de producir heno, pescar en los estanques locales, y comerciar productos de sus propios terrenos. El 5 de agosto, después de que sólo un 8 por ciento de los trabajadores de los Urales se ofreciera para unirse al Ejército Rojo, los bolcheviques anunciaron el reclutamiento obligatorio, llevando a que el sindicato, dominado por eseristas veteranos, con el

11. Este ensayo no contempla los trabajos recientes sobre la oposición socialista y anarquista a los bolcheviques. Sin embargo, se debe hacer mención a la excelente iniciativa de la editorial Rosspen, que continuó publicando la serie sobre los partidos políticos en Rusia, poniendo a disposición una masa de documentación. Los cuatro volúmenes que se mencionan a continuación, editados por Ziva Galili y A.P. Nenarokov, se publicaron en Moscú por Rosspen, y la mayoría fueron discutidos en un foro de opinión, "Historia documental y partidos políticos", Kritika, 5, 1, 2004, pp. 107-232: Men'sheviki v 1918 godu (1999); Men'sheviki v 1919-1920 gg. (2000); Men'sheviki v Rossii bol'shevistkoi 1921-1922 (2002); y Men'sheviki $v$ bol'shevistskoi Rossii, 1922-1924 gg. (2004). Otros volúmenes de la serie incluyen N.D. Erofeev (ed.), Partiia sotsialistov-revoliutsionerov: Dokumenty i materialy, 1900-1925, 3 vols., esp. 3, parte 1: Fevral'-oktiabr' 1917 g. (2000); I. y V. Leonti'ev (eds.), Partiia levykh sotsialistov-revoliutsionerov: Dokumenty i materialy, 1: 1917 Iiul' G.-mai 1918 g. (2000); V.V. Kriven'kii (ed.), Anarkhisty: Dokumenty i materialy, 2: 1917-1935 gg.(1999); B. y D. Pavlov (eds.), Soiuz eserov-maksimalistov: Dokumenty $i$ publitsistika, 1906-1924 gg. (2002). Dos trabajos secundarios interesantes sobre los eseristas son los de Scott B. Smith (2011) y Dobrovol'skii (2002). 
apoyo de los trabajadores, tomara el control de la ciudad (Postnikov y M. A. Fel'dman, 2009: 313). ${ }^{12}$ Miles de trabajadores se unieron al Ejército Popular, que fue finalmente sometido a mediados de noviembre por el Segundo Ejército Rojo. Ya en 1982 M. S. Bernshtam había publicado documentación sobre el levantamiento, pero los trabajos más recientes ofrecen muchos más detalles y una mejor impresión sobre el modo en que la fuerza de trabajo en las fábricas de municiones se hallaba dividida entre un proletariado hereditario, poseedor de tierras, y aquellos que habian entrado en las fábricas durante la guerra (Bernshtam, 1982; Fel'dman, 2012; Korobeinikov, 2013).

También persiste la fascinación de los historiadores rusos por el movimiento blanco. Hasta el momento se han publicado diecisiete volúmenes de la serie Beloe dvizhenie v Rossii (El Movimiento Blanco en Rusia), un proyecto inspirado por el deseo de superar la cruda tendenciosidad de la historiografia soviética y afrontar el tema en forma desapasionada. ${ }^{13}$ No puede decirse que toda la serie lo consiga: algunos estudiosos, en sintonía con el actual recrudecimiento del nacionalismo, tratan a los blancos como los nobles defensores del Estado ruso, traicionado por masas cosmopolitas bolcheviques y anárquicas. Los trabajos más sobrios revelan cómo el Ejército Voluntario, una vez fuera de su núcleo en las regiones cosacas, tenía los mismos problemas que sus rivales a la hora de movilizar mano de obra y recursos (Gagkuev, 2012). El Ejército Voluntario no habria tenido nada que ver con la "contrarrevolución democrática" -es decir, el intento de los eseristas por restaurar la Asamblea Constituyente-, mientras que en los Urales y en Siberia, las fuerzas blancas tardaron casi un año en volverse contra sus aliados más moderados (Shishkin, 2007).

En general, los trabajos recientes subrayan la complejidad de las fuerzas que se enfrentaron en la guerra civil, en la cual bolcheviques, ejércitos blancos rivales, fuerzas nacionalistas de Ucrania, Georgia y otras regiones, verdes y anarquistas luchaban por controlar el territorio y los recursos humanos y materiales. En Ucrania, el entramado de elementos nacionales, de clase e ideológicos era particularmente complejo, como lo revela un detallado estudio de V.F. Soldatenko (2012). Stephen Velychenko (2010) compara el modo en que las fuerzas rivales en Ucrania lucharon para construir un estado. En su último estudio sobre la guerra civil en el norte de Rusia, Liudmila Novikova (2011) investiga cómo

12. Los autores estiman que al menos una cuarta parte de los trabajadores de las empresas estatales de los Urales apoyó a los blancos.

13. Beloe dvizhenie $v$ Rossii (Moscú: Tsentrpoligraf). Se han programado al menos otros cuatro volúmenes. Para obtener una lista actual, consultar www.centrpoligraf. $\mathrm{ru} /$ series. . php?page $=204$. 
el pueblo respondió a los esfuerzos de los blancos para administrar el territorio, así como a las operaciones de las fuerzas expedicionarias de la Entente. En general, está claro que el conjunto de las fuerzas rivales enfrentó los mismos problemas a la hora de movilizar recursos para sostener operaciones militares y crear una administración duradera frente a la resistencia de las bases a las requisas y el reclutamiento, y la falta de voluntad de los combatientes para luchar más allá de su territorio inmediato (Butakov, 2000). A pesar de la abundancia de nuevos detalles -que incluyen evidencias del apoyo popular, así como de la clase media, a los blancos- el cuadro general que emerge de la guerra civil no es muy distinto del que fuera diseñado por los historiadores occidentales en las décadas de 1980 y 1990.

Por último, continúan apareciendo trabajos sobre la iglesia ortodoxa. En la década de 1990, el tenor de la mayor parte de éstos era hagiográfico: la iglesia era presentada simplemente como la víctima de un régimen despiadado, que luchaba por mantener la neutralidad frente a un hostigamiento sin tregua. Los mejores trabajos publicados desde 2000 han mejorado este tono: M.A. Babkin (2007) discute el rápido abandono eclesiástico a la monarquía después de febrero, y la desorientación, la división y la descentralización del poder que vino a continuación. P.G. Rogoznyi (2008) examina el papel de los obispos y la "revolución de la iglesia" que siguió a los esfuerzos para elegir al clero en todos los niveles. El alcance del apoyo eclesiástico a los blancos sigue siendo poco claro. En un estudio imparcial sobre la iglesia en los Urales, una zona de importantes conflictos en la guerra civil, M.G. Nechaev contabiliza 78 casos de resistencia al decreto que separaba a la Iglesia del Estado; cuatro casos de negativa a entregar los registros de la iglesia; 18 casos de clérigos "que dan su bendición" a acciones armadas contra el régimen bolchevique; y cuatro casos de sacerdotes que participaron en actividades clandestinas (2004: 204). También revela la ferocidad del terror rojo en los Urales. El arzobispo Andronnik, de la diócesis de Perm, encontró una muerte particularmente horrible a manos de la Cheka el 20 de junio de 1918, por haber ordenado a los clérigos que suspendieran todos sus servicios. Al mismo tiempo, a pesar de que los niveles de represión contra clérigos sospechosos de estar implicados en el movimiento blanco fueron mayores en los Urales que en cualquier otra parte, las victimas estaban todavía lejos de ser tan numerosas como suponian muchas de las estimaciones generalizadas en la década de 1990. En el transcurso de 1918, parece que hubo 101 clérigos en la diócesis de Perm, quince en Orenburg, y al menos 44 en Ekaterinburg que fueron asesinados por las fuerzas bolcheviques. ${ }^{14} \mathrm{En}$

14. P.I. Kostogryzov, “Uchastie Pravoslavnoi tserkvi v antibol'shevistskom sopro- 
Voronezh, en 1919, donde los conflictos con la iglesia fueron también feroces, fueron asesinados 160 clérigos. ${ }^{15}$ Las estimaciones del número de religiosos muertos por todo el imperio varian de 827 sacerdotes y monjes fusilados en 1918 y 19 en 1919 (junto con 69 presos), a 3.000 clérigos asesinados y 1.500 sujetos a castigo en 1918, y 1.000 fusilados y 800 sujetos a castigo en 1919. ${ }^{16}$

Como espero que este estudio haya puesto de relieve, el trabajo historiográfico desde el comienzo del nuevo siglo ha ampliado en gran medida nuestro conocimiento sobre muchos aspectos de la revolución. Es más discutible que este mayor conocimiento haya mejorado nuestra comprensión de los procesos. En la actualidad, los historiadores son más propensos a ver la revolución como el inicio de un ciclo de violencia que condujo inexorablemente a los horrores del estalinismo y el nazismo que a entenderla como un intento imperfecto para crear un mundo mejor. Son más propensos a entender la movilización de masas como motivada por el irracionalismo y la agresión antes que por la indignación ante la injusticia o por el anhelo de libertad. Este punto de vista se deriva, sin duda, de la sensación correcta de que la Revolución Rusa fue un fracaso y de que las revoluciones del siglo XX en general tendieron a producir regímenes peores que los que derrocaron. Sin embargo, en el mundo contemporáneo existen otros factores más sutiles que también dan forma a nuestras percepciones de la revolución. Vivimos en un mundo en el cual el discurso (históricamente muy reciente) de los derechos humanos, admirable en todo tipo de formas, ha desempeñado un papel para marginalizar valores tales como la justicia distributiva, la igualdad socioeconómica o el bien común, que siguieron formando parte de las principales corrientes políticas, al menos en Europa, a lo largo de la Guerra Fría, y que parecian tener algún eco, si bien distante, con los valores de 1917. Tenemos la suerte, también, de vivir en un mundo (occidental) donde, en una medida que no tiene precedentes históricos, estamos en gran parte aislados de la violencia (Bessel, 2015). Son factores que, de manera subliminal, tienden a influenciar la manera en que percibimos la revolución rusa en el siglo XXI: por un lado, nos sensibilizan sobre los terribles costos de una transformación revolucionaria; por el otro,

tovleniie v 1917-1918 gg. (na materialakh Urala)" (www.dk1868.ru/statii/pavel) Pravosl.htm).

15. V.V. Viatkin, "Pervyi period deistviia administrativno-silovoi modeli gosudarstvennotserkovnykh otnoshenii na Urale (oktiabr' 1917-pervaia polovina 1919 g.)”, www.permgani. ru/publikatsii/konferentsii/grazhdanskaya-vojna-na-vostoke-rossii/ $v$ v-vyatkin-pervyj-perioddejstviya- administrativno-silovoj-modeli-gosudarstvennotserkounyh-otnoshenij-na-urale. html.

16. Dmitrii Sokolov, "Russkaia pravoslavnaia tserkov'v period gonenii (1917-1937 gg.)", www. rusk.ru/st.php?idar=112187. 
limitan nuestra comprensión intelectual e imaginativa sobre las causas que generan las revoluciones y, en concreto, respecto a la Revolución Rusa, sobre qué llevó a que el ideal de la sociedad socialista fuera tan atractivo para millones de personas. Advertimos con bastante facilidad la violencia y el derramamiento de sangre, pero nos resulta más dificil advertir el idealismo, la esperanza y el auto-sacrificio que también fueron componentes claves de la revolución. No hay dudas de que los historiadores tienen la responsabilidad de ocuparse de la violencia, la agresión, y el sufrimiento, pero reducir la Revolución Rusa a estas cuestiones da como resultado una historia empobrecedora. ${ }^{17} \mathrm{~A}$ medida que avanzamos hacia el centenario de la Revolución Rusa, entonces, debemos tratar de comprender cómo la pobreza y el atraso económico, la indiferencia de los privilegiados y la disposición de los Estados a utilizar su fuerza represiva son factores que ayudaron a generar la violencia que deploramos. La responsabilidad que recae sobre nosotros es capturar las contradicciones de la Revolución Rusa y escribir su historia en el modo de una tragedia en lugar de un cuento de tono moralizante.

\section{Bibliografia}

Baberowski, Jörg (2012), Verbrannte Erde: Stalins Herrschaft der Gewalt, Munich: Beck.

Babkin, M.A. (2007), Dukhovenstvo Russkoi pravoslavnoi tserkvi i sverzhenie monarkhii, nachalo XX v.-konets $1917 \mathrm{~g}$., Moscú: Gosudarstvennaia publichnaia istoricheskaia biblioteka Rossii.

Badcock, Sarah (2007), Politics and the People in Revolutionary Russia: A Provincial History, Cambridge: Cambridge University Press.

Bernshtam, M.S. (ed.) (1982), Narodnoe soprotivlenie kommunizmu: Ural $i$ Prikam'e, noiabr' 1917-ianvar' 1919 gg., París: YMCA Press.

Bessel, Richard (2015), Violence: A Modern Obsession, Nueva York: Simon and Schuster.

Beyrau, Dietrich (2003), "Der Erste Weltkrieg als Bewährungsprobe: Bolschewistische Lernprozesse aud dem 'imperialistischen' Krieg”, Journal for Modern European History, 1, 1, pp. 96-123.

Bibin, M.A. (2000), Dvorianstvo nakanune padeniia tsarizma $v$ Rossii, Saransk: Saranskii kooperativnyi institute.

Buldakov, V.P. (1997), Krasnaia smuta: Priroda i posledstviia revoliutsionnogo nasiliia, Moscú: Rosspen.

- (2007), Quo vadis? Krizisy v Rossii: Puti pereosmysleniia, Moscú: Rosspen.

- (2010), Khaos i etnos: Etnicheskie konflikty v Rossii, 1917-1918, Moscú: Novyi khronograf.

17. Comparar con la interpretación de Simon Schama (1989: xv) sobre la Revolución Francesa: "en cierto sentido inevitable y deprimente, la violencia era la revolución". 
Butakov, I.A. (2000), Beloe dvizhenie na Iuge Rossii: Kontseptsiia i praktika gosudarstvennogo stroitel'stva (konets 1917-nachalo 1920 g.), Moscú: RUDN.

Churakov, D.O. (2004), Revoliutsiia, gosudarstvo, rabochii protest: Formy, dinamika i priroda massovykh vystuplenii rabochikh $v$ Sovetskoi Rossii, 1917-1918 gg., Moscú: Rosspen.

Dobrovol'skii, A. V. (2002), Ésery v Sibiri vo vlasti i v Oppozitsii (1917-1923 gg.), Novosibirsk: Novosibirskii gosudarstvennyi Universitet.

DuGarm, Delano (2001), "Local Politics and the Struggle for Grain in Tambov, 1918-1921", en Provincial Landscapes: Local Dimensions of Soviet Power, 1917-1953, Pittsburgh: University of Pittsburgh Press.

Fatueva, N.V. (1996), Protivostoianie: Krizis vlasti-tragediia naroda, Riazan': Rus'.

Fel'dman, M.A. (2012), “Izhevsko-votkinskoe rabochee vosstanie skvoz' prizmu sotsial'noi istorii," Rossiiskaia istoriia, $\mathrm{n}^{\circ} 3$, pp. 12-20.

Figes, Orland y Boris Kolonitskii (1999), Interpreting the Russian Revolution: The Language and Symbols of 1917, New Haven: Yale University Press.

Frame, Murray, Boris Kolonitskii, Steven G. Marks y Melissa K. Stockdale (eds.) (2014), Russian Culture in War and Revolution, 1914-1922, 2 vols., Bloomington: Slavica Publishers.

Fuller, William C. Jr. (2006), The Foe Within: Fantasies of Treason and the End of Imperial Russia, Ithaca: Cornell University Press.

Gagkuev, R.G. (2012), Beloe dvizhenie na Iuge Rossii: Voennoe stroitel'stvo, istochniki komplektovaniia, sotsial'nyi sostav, 1917-1920 gg., Moscú: Posev.

Gatrell, Peter (2005), "Prisoners of War on the Eastern Front during World War I," Kritika, 6, 3, págs. 557-566.

Gerasimenko, G.A. (1972), Sovety Nizhnego Povol'zhia v Oktiabr'skoi revoliutsii, Saratov: Izdatel'stvo Saratovskogo universiteta.

Grunt, A.A. (1976), Moskva,1917-yi: Revoliutsiia i kontrrevoliutsiia, Moscú: Akademiia nauk SSSR.

Hickey, Michael C. (1996), "Peasant Autonomy, Soviet Power, and Land Redistribution in Smolensk Province, November 1917-May 1918", Revolutionary Russia, 9, 1, pp. 19-32.

Hoffmann, David L. (2011), Cultivating the Masses: Modern State Practices and Soviet Socialism, 1914-1939, Ithaca: Cornell University Press.

- y Yanni Kotsonis (eds.) (2000), Russian Modernity: Politics, Knowledge, Practices, Basingstoke: Macmillan.

Holquist, Peter (2002), Making War, Forging Revolution: Russia's Continuum of Crisis, 1914-1921, Cambridge, MA: Harvard University Press.

- (2003), "Violent Russia, Deadly Marxism? Russia in the Epoch of Violence, 1905-1921”, Kritika, 4, 3, pp. 627-652.

Hosking, Geoffrey (1997), Russia: People and Empire, 1552-1917, Londres: Harper Collins.

Iarov, S.V. (1999), Krest'ianin kak politik: Krest'ianstvo Severo-Zapada Rossii v 1918-1919 gg., San Petersburgo: RAN. 
- (2006), Konformizm v Sovetskoi Rossii: Petrograd, 1917-1920 gg., San Petersburgo: Evropeiskii dom.

Kir'ianov, I.I. (2001), Pravye partii v Rossii, 1911-1917, Moscú: Rosspen.

Kolonitskii, B.I. (2001), Simvoly vlasti i bor'ba za vlast': K izucheniiu politicheskoi kul'tury rossiiskoi revoliutsii, San Petersburgo: Dmitrii Bulanin.

- (2010), "Tragicheskaia erotika": Obrazy imperatorskoi sem'i v gody Pervoi mirovoi voiny, San Petersburgo: Novoe literaturnoe obozrenie.

Kondrashin, V.V. (2001), Krest'ianskaia vandeia v Povolzh'e 1918-1921 gg., Moscú: Ianus-K.

Korobeinikov, A.V. (2013), Votkinskaia Narodnaia armiia v 1918 g., pt. 1, Izhevsk: Idnakar.

Kostogryzov, P.I. "Uchastie Pravoslavnoi tserkvi v antibol'shevistskom soprotovleniie v 1917-1918 gg. (na materialakh Urala)” www.dk1868.ru/ statii/pavel/Pravosl.htm.

Kovalev, D.V. (2007), “Sotsializatsiia zemli i krest'ianskoe zemlepol'zovanie," Rossiiskaia istoriia, $\mathrm{n}^{\circ}$ 5, pp. 97-106.

Landis, Erik (2008), Bandits and Partisans: The Antonov Movement in the Russian Civil War, Pittsburgh: University of Pittsburgh Press.

Lohr, Eric (2003), Nationalizing the Russian Empire: The Campaign against Enemy Aliens during World War I, Cambridge: Harvard University Press.

-, Vera Tolz, Alexander Semyonov y Mark von Hagen (eds.) (2014), The Empire and Nationalism at War, Bloomington: Slavica Publishers.

Lyandres, Semion (2013), The Fall of Tsarism: Untold Stories of the February 1917 Revolution, Oxford: Oxford University Press.

Mayer, Arno (2001), The Furies: Violence and Terror in the French and Russian Revolutions, Princeton: Princeton University Press.

Morozova, O.M. (2010), Dva akta dramy: Boevoe proshloe i poslevoennaia pousednevnost' veteranov grazhdanskoi voiny, Rostov-on-Don: Iuzhnyi nauchnyi tsentr Rossiiskoi akademii nauk [RAN].

- (2012), Antropologiia grazhdanskoi voiny, Rostov-on-Don: Iuzhnyi nauchnyi tsentr RAN.

Nagornaia, O. S. (2010), Drugoi voennyi opyt: Rossiiskie voennoplennye Pervoi mirovoi voiny v Germanii (1914-1922), Moscow: Novyi khronograf.

Narskii, I. V. (2001), Zhizn'v katastrofe: Budni naseleniia Urala v 1917-1922 gg., Moscú: Rosspen.

Narskii, I.V. et al. (eds.) (2011), Slukhi $v$ istorii Rossii XIX-XX vekov: Neformal'naia kommunikatsiia i krutye povoroty rossiiskoi istorii, Cheliabinsk: Kamennyi poias.

Naumov, V.P. y A.A. Kosakovskii (eds.) (1997), Kronshtadt 1921: Dokumenty, Moscú: Rossiia XX vek.

Nechaev, M.G. (2004), Tserkov' na Urale v period velikikh potriasenii, 19171922, Perm: Ural'skii gosudarstvennyi universitet.

Novikova, L.G. (2011), Provintsial'naia "kontrrevoliutsiia": Beloe dvizhenie i Grazhdanskaia voina na russkom Severe, 1917-1920, Moscú: Novoe literaturnoe obozrenie. 
Penter, Tanja (2000), Odessa 1917: Revolution an der Peripherie, Colonia: Böhlau.

Plaggenborg, Stefan (1996), "Gewalt und Militanz in Sowjetrußland 19171930”, Jahrbücher für Geschichte Osteuropas 44, págs. 409-430.

Porshneva, Ol'ga (2013), "Vlast' i rabochie Urala: Evoliutsiia vzaimootnoshenii v usloviiakh Grazhdanskoi voiny", Rossiiskaia istoriia, n ${ }^{\circ}$, pp. 47-62.

Posadskii, Anton (2010), Ot Tsaritsyn do Syzrani: Ocherki grazhdanskoi voiny na Volge, Moscú: AIRO-XXI.

Postnikov, S.P. y M.A. Fel'dman (2009), Sotsiokul'turnyi oblik promyshlennykh rabochikh Rossii v 1900-1941 gg., Moscú: Rosspen.

Rabinowitch, Alexander (2007), The Bolsheviks in Power: The First Year of Soviet Rule in Petrograd, Bloomington: Indiana University Press.

Raleigh, Donald J. (1986), Revolution on the Volga: 1917 in Saratov, Ithaca: Cornell University Press.

- (2002), Experiencing Russia's Civil War: Politics, Society, and Revolutionary Culture in Saratov, 1917-1922, Princeton: Princeton University Press.

Rendle, Matthew (2009), Defenders of the Motherland: The Tsarist Elite in Revolutionary Russia, Oxford: Oxford University Press.

Retish, Aaron (2008), Russia's Peasants in Revolution and Civil War: Citizenship, Identity, and the Creation of the Soviet State, 1914-1922, Cambridge: Cambridge University Press.

Retish, Aaron, Sarah Badcock y Liudmila Novikova (eds.) (2015), Russia's Home Front in War and Revolution, 1914-1922, Bloomington: Slavica Publishers.

Rogoznyi, P.G. (2008), Tserkovnaia revoliutsiia 1917 goda, San Petersburgo: Liki Rossii.

Sablin, V.A. (2009), Krest'ianskoe khoziaistvo na Evropeiskom Severe Rossii (1917-1920), Moscú: Academia.

Sanborn, Joshua A. (2003), Drafting the Russian Nation: Military Conscription, Total War, and Mass Politics, 1905-1925, DeKalb: Northern Illinois University Press.

- (2014), Imperial Apocalypse: The Great War and the Destruction of the Russian Empire, Oxford: Oxford University Press.

Schama, Simon (1989), Citizens: A Chronicle of the French Revolution, Nueva York: Knopf.

Schnell, Felix (2012), Räume des Schreckens: Gewalt und Gruppenmilitanz in der Ukraine, 1905-1933, Hamburg: Hamburger Edition.

Shishkin, V.I. (ed.) (2000), Sibirskaia Vandeia, 1: 1919-1920, Moscú: Mezhdunarodnyi fond "Demokratiia".

- (ed.) (2007), Vremennoe sibirskoe pravitel'stvo, 26 maia-3 noiabria 1918 g.: Sbornik dokumentov i materialov, Novosibirsk: Sova.

Smith, Douglas (2012), Former People: The Last Days of the Russian Aristocracy, Basingstoke: Palgrave Macmillan.

Smith, Stephen A. (1994), "Writing the History of the Russian Revolution after the Fall of Communism", Europe-Asia Studies, 4, 4, pp. 563-578. 
- (2006), "The Revolutions of 1917-1918," en The Cambridge History of Russia, 3: The Twentieth Century, Cambridge: Cambridge University Press.

- (2008), Revolution and the People in Russia and China: A Comparative History, Cambridge: Cambridge University Press.

Smith, Scott B. (2011), Captives of Revolution: The Socialist Revolutionaries and the Bolshevik Dictatorship, 1918-1923, Pittsburgh: University of Pittsburgh Press.

Soldatenko, V.F. (2012), Grazhdanskaia voina v Ukraine, 1917-1920 gg., Moscú: Novyi khronograf.

Steinberg, Mark D. (2001), Voices of Revolution, 1917, New Haven: Yale University Press.

Velychenko, Stephen (2010), State Building in Revolutionary Ukraine: A Comparative Study of Government and Bureaucrats, 1917-1922, Toronto: University of Toronto Press.

Vinogradov, V.K. y V.P. Kozlov (eds.) (1999), Kronshtadtskaia tragediia 1921 goda: Dokumenty, 2 vols., Moscú: Rosspen.

$$
* * *
$$

Título: "The Historiography of the Russian Revolution 100 Years On."

Resumen: $\mathrm{El}$ artículo ofrece un análisis del modo en que la historiografia reciente ha contribuido a nuestra comprensión sobre la Revolución Rusa, identificando las tendencias y problemas analíticos que han ocupado a los historiadores desde, aproximadamente, los inicios del siglo XXI. Comienza con un bosquejo de ciertas tendencias interpretativas que han tenido influencia en la historiografia reciente y luego rastrea algunos de los temas que han atraído el mayor interés académico. Palabras clave: Revolución Rusa - guerra civil - violencia - bolcheviques

Abstract: The article provides an assessment on how recent historiography has shaped our understanding of the Russian Revolution by identifying the trends and analytical issues that have exercised historians since roughly the beginning of the 21 st century. It begins by sketching certain interpretive trends that have been influential in recent historiography and goes on to map some of the topics that have attracted the most scholarly interest.

Palabras clave: Russian Revolution - civil war - violence - bolsheviks 